\title{
INVENTAIRE PISCICOLE, EN 1960, DES LACS DE MONTAGNE DES BASSES ET HALTES-PYRENÉES
}

\author{
Par P. CHIMITS, \\ Ingénieur des Eaux et Forets.
}

Nous avons eu l'occasion de publier un inventaire piscicole des lacs de montagne des Hautes et Basses-Pyrénées à la date de 1952, ainsi que deux articles sur le peuplement de ces lacs de montagne.

Il nous semble opportun de faire le point, à la date de août 1960 , de cet inventaire piscicole en tenant compte des repeuplements effectués depuis 1952.

A l'inventaire des lacs publié en 1952, nous devons retrancher le lac de Loustallat dans la vallée de la Neste, qui a disparu, absorbé par le grand lac de barrage de Capdelong, et ajouter 5 lacs-barrages nouveaux créés par E. D. F. et S. N. C. F. et qui ont d'ailleurs été alevinés.

Nous rappelons brièvement que la Truite commune était, il y a vingt-cinq ans encore, la seule espèce autochtone peuplant une partie de ces lacs, la plupart d'entre eux étant vierges. Le premier essai de repeuplement artificiel a été fait, en 1906, par M. Paul Descombes, Président de l'Association Centrale pour l'Aménagement des montagnes, qui fit transporter $\mathbf{5 0 0}$ alevins de Truites arc-en-ciel dans le lac de Barroudé, dans la vallée de la Neste. Il n'en reste plus trace aujourd'hui. Nous ne trouvons ensuite aucune trace de repeuplement jusqu'en 1936. A cette époque, M. LARrieu, alors Ingénieur principal des Eaux et Forêts à Oloron (dont nous étions l'adjoint), exécuta un important travail d'aménagement piscicole dans le Sud-Ouest consistant, notamment, en ce qui concerne les Hautes et Basses-Pyrénées, en la mise en service d'établissements de pisciculture (Lées-Athas, Cauterets, Argelès et Bagnères). Avec les alevins produits par ces établissements et par l'ancien établissement d'Oloron, M. LARrieu entreprit un travail de peuplement et d'introduction de ces nouvelles espèces dans les lacs de montagne, ajoutant à la Truite commune : l'Omble chevalier, la Truite arc-en-ciel et le Saumon de fontaine. Ce travail fut interrompu par la guerre de 1939. Nous pouvons constater aujourd'hui que, dans la plupart des lacs alevinés à cette époque, le peuplement introduit s'est fort bien maintenu, malgré une pêche parfois assez intense. L'Omble chevalier en particulier, s'est remarquablement acclimaté et reproduit, même dans les lacs de faible profondeur, alors que dans les lacs alpins, sa station d'origine, l'Omble 
chevalier est considéré comme une espèce de grands fonds. Il donne souvent, dans ces lacs pyrénéens peu profonds, des populations naines (lac d'Oncet et lac d'Isabe). En revanche, dans les lacs profonds, on trouve des individus de belle taille (lac de Miguelou). Une particularité de la biologie de l'Omble chevalier pyrénéen est également à signaler : alors que l'Omble chevalier, dans les lacs alpins, ne quitte pas les grands fonds, l'Omble dans les lacs pyrénéens, peut monter en surface et moucheronner. C'est ainsi qu'en juin 1958, au lac de Gréziolles, ayant aperçu des gobages nombreux, je montai ma canne à mouche et capturai 31 Ombles chevaliers et 3 Truites en moins de 3 heures.

A partir de 1950, nous avons été chargé du service de Restauration des Terrains en Montagne pour les Hautes et Basses-Pyrénées. La gestion de la pisciculture de Cauterets incombe à ce service. Elle était vide de poissons en 1950. Nous l'avons remise en état et créé des bassins pour stocker les géniteurs. Cet établissement, avec son annexe de Sassis, créée en 1954, contient maintenant un important cheptel de géniteurs des 4 éspèces ci-dessus et de Cristivomer namaycush, nouvelle espèce américaine. Il produit actuellement environ 2.000.000 oufs de salmonides par an qui sont en partie expédiés dans des établissements domaniaux des Alpes, du Massif Central et des Pyrénées, mais dont ceux qui restent ont permis chaque année un effort local de repeuplement. A ces espèces de salmonides, s'ajoute la Tanche qui semble donner de bons résultats dans certains lacs de moindre altitude et de faible profondeur (Laquette de Barèges). C'est ainsi que le Service Forestier a pu, depuis 1950, entreprendre un effort constant de repeuplement de ces lacs de montagne, portant sur une douzaine de lacs nouveaux en moyenne chaque année et sur l'entretien des repeuplements précédents.

Les résultats de dix ans de travail sont consignés dans l'inventaire ci-après et sont à porter entièrement à l'actif du Service Forestier, aidé occasionnellement par quelques volontaires, à la seule exclusion des 3 lacs de la vallée de Barada, alevinés en 1957 par la Société des Pêcheurs de Tarbes.

Il convient de préciser que, jusqu'en 1957, tous ces alevinages ont été faits à dos d'homme ou de mulet, et, le cas échéant, par téléférique, les alevins étant transportés dans des bidons ou des sacs en plastique, avec ou sans aérateur d'air ou diffuseur d'oxygène. Tous ces alevinages avaient obligatoirement lieu en Juin ou début de Juillet, lorsque les lacs cessent d'être glacés et avant les trop fortes chaleurs. Les alevins étaient donc, par conséquent, toujours âgés de 3 à 4 mois environ. Les résultats qu'ils ont donnés dans ces lacs ont été excellents. Il faut compter, pour un lac d'altitude moyenne (2.000 à $2.200 \mathrm{~m}$.), environ 3 heures de marche et 20 à $30 \%$ de mortalité pendant le transport.

\section{Hélicoptère et lacs de montagne.}

L'hélicoptère est employé depuis assez longtemps, pour l'alevinage des lacs isolés, par les services de la pêche des Êtats-Unis et du Canada. En France, des alevinages par hélicoptère ont été faits, en 1956 dans 
l'Isère et en 1957 dans l'Ariège. Mais, dans ces deux cas, Ariège et Isère, les hélicoptères devaient se poser à proximité des lacs à aleviner et les passagers devaient porter les bidons contenant les alevins jusqu'au lac. D'autre part, des gardes devaient monter à l'avance pour repérer un terrain d'atterrissage de fortune. Enfin, l'atterrissage et l'arrêt occasionnaient des pertes de temps extrêmement coûteuses en raison de l'immobilisation de l'hélicoptère dont l'heure est actuellement facturée à plus de 500 NF. Ces opérations sont sans doute spectaculaires, mais peu rentables.

C'est la raison pour laquelle j'ai procédé, depuis Juin 1957, à des essais d'alevinage en vol afin de réduire au minimum les temps morts de l'utilisation de l'hélicoptère et d'essayer de définir une méthode économique.

Au printemps 1957, des essais préalables de jet en bassin de sacs en plastique contenant 8 litres d'eau et 500 alevins de 3 mois à partir de hauteurs variant entre 3 et 5 mètres. se montrèrent favorables. Les sacs s'enfonçaient lentement dans l'eau du bassin, celle-ci pénétrant par l'ouverture du sac et les alevins quittant le sac les uns après les autres. Les pertes dues au choc ou à l'emprisonnement des alevins dans les replis du plastique, étaient de l'ordre de grandeur de $10 \%$.

Le 3 Juillet 1957, deux hélicoptères partirent de la base de Pau, avec une charge de sacs en plastique contenant chacun 500 alevins qu'ils avaient pour consigne de larguer de 3 à 5 mètres de haut dans les lacs de la haute vallée du Valentin. Il fut impossible de les larguer de cette faible hauteur en raison de fortes turbulences d'air existant en plein été à la surface de ces lacs de montagne très encaissés. Le seul point acquis de cette expérience était qu'il est dangereux en hélicoptère de passer à moins de 5 mètres de la surface des lacs de montagne.

En Février 1958, j'eus l'occasion de rencontrer le Chef des Services de la Pêche Canadiens, M. Louis Pruvost, qui fnous confirma que ses services larguaient des Truitelles en vol dans les lacs, sans récipient et à des hauteurs variant de 30 à 50 mètres. Aussi, au printemps 1958, des essais de larguage directs eurent lieu dans le bassin d'alevinage du lac d'Uzein, près de Pau, situé à proximité immédiate de la base d'hélicoptères. Les deux portes de l'hélicoptère étant enlevées, il fut procédé au lancement, sans récipient, aux trois hauteurs de 20,40 et 60 mètres, d'abord de 6 'Truites arc-en-ciel adultes, puis d'une cinquantaine de Truites arc-en-ciel de 12 à 15 centimètres. Nous n'enregistrâmes aucune perte. Je procédai ensuite au renversement depuis l'hélicoptère, toujours portes enlevées, et toujours des trois hauteurs de 20,40 et 60 mètres, de 6 seaux contenant chacun 100 alevins de Truites arc-en-ciel de 3 mois. Au point d'impact de ces six "pluies d'alevins " il fut retrouvé des alevins morts, au nombre de 4 à 9 par point de chute. Le pourcentage des pertes était donc de 4 à $9 \%$, bien inférieur aux pertes normales d'un transport de 2 à 3 heures à dos d'homme ou de mulet. Les alevins ainsi tombés en pluie se montraient vigoureux et, dans les jours qui suivirent, aucune perte ne fut constatée. Dès lors, il était possible de tenter l'essai en montagne. 
Précisément, une société auxiliaire de la S. N. C. F., la S. C. E. T. A. s'installait à Cauterets cet été pour le transport de touristes en montagne et disposait d'hélicoptères Bell $47 \mathrm{~J}$. Après autorisation de l'Administration, il fut convenu avec cette société d'effectuer un premier essai de transport d'alevins dans les lacs de l'Ardiden situés à 2.400 mètres d'altitude en partant de Cauterets à l'altitude 900 mètres. L'hélicoptère Bell, piloté par M. Morel, et me portant comme passager, avec 4 bidons

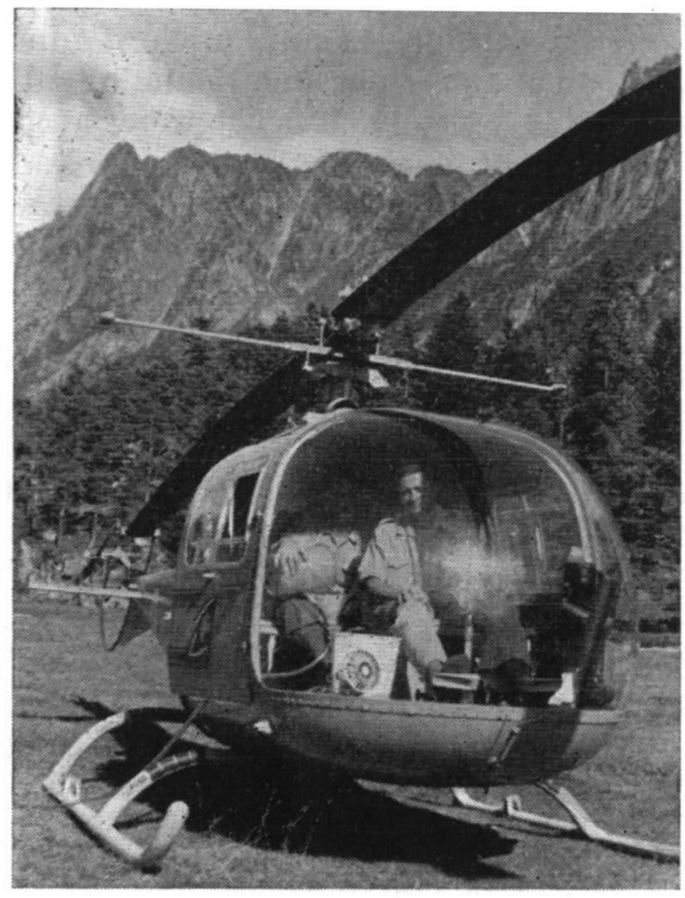

Système de déversement en vol.

Le passager renverse le seau d'alevins dans l'entonnoir

les alevins sont conduits par un tuyau en plastique au-dessous des patins d'atterissage.

d'alevins, partit de bonne heure afin de profiter des ascendances le long des parois, l'altitude de $\mathbf{2 . 4 0 0}$ mètres étant voisine de la limite supérieure de vol de l'hélicoptère. Nous avons pu ainsi aleviner dans de bonnes conditions les 4 principaux lacs de l'Ardiden.

Un dernier dispositif pratique fut alors mis au point. Le renversement des seaux d'alevins nécessite en effet l'enlèvement des portes de l'hélicoptère ce qui, en haute montagne, peut impressionner le passager. D'autre part, au cours des renversements, certains alevins heurtent les patins de l'hélicoptère et des souffles de vent peuvent rabattre les alevins contre l'hélicoptère lui-même. Aussi avons-nous finalement mis au point le dispositif suivant : par un trou du plancher de l'hélicoptère passe un tuyau de $2 \mathrm{~m}$. 50 de long, d'un diamètre de 33 millimètres avec, à l'extrémité supérieure, un entonnoir, l'extrémité inférieure descendant un peu en dessous des patins. Le passager, arrivé au-dessus du lac, se contente 
de déverser par l'entonnoir le contenu d'un seau d'alevinage lequel descend par le tuyau en dessous des patins et tombe en pluie sur le lac. L'essai fait dans ces conditions au lac du Paradis à Cauterets par 30 mètres de hauteur environ, se montra satisfaisant et il ne fut pas constaté de perte.

Ainsi, un système pratique et économique de déversement d'alevins dans les lacs de montagne est-il mis au point. Le matériel est peu coûteux (2 m. 50 de tuyau plastique de $33 \mathrm{~mm}$., un entonnoir et des seaux). D'autre part, les lacs pyrénéens se trouvent tous ou presque tous entre 1.800 et 2.500 mètres d'altitude. Il suffit donc d'approvisionner à une base de départ, le plus haut possible, une bonne quantité d'alevins dont un garde surveillera l'état de santé. L'hélicoptère, avec un seul passager et le dispositif ci-dessus peut, en un seul vol préparé à l'avance, aleviner un groupe de 4 à 5 lacs situés à des altitudes très voisines et revenir quelques minutes après au réapprovisionnement à sa base pour desservir de suite après, un nouveau groupe de lacs voisins à altitudes similaires. Ainsi, les pertes de temps et de surconsommation de carburant sont évitées. En 1 heure de vol, compte tenu du retour à la station de départ et du temps perdu pour le rechargement, c'est-à-dire pour une dépense de 50 à 55.000 francs, l'hélicoptère peut aleviner en vol une bonne quinzaine de lacs au lieu de 2 ou 3 s'il doit se poser. Il est dès lors possible, dans les Hautes et Basses-Pyrénées, d'aleviner chaque année une trentaine ou une quarantaine de lacs, alors qu'il était impossible d'en aleviner plus d'une douzaine.

L'alevinage par hélicoptère des lacs de montagne est donc devenu une opération, pas plus onéreuse que le système classique de transport par porteur ou mulet, mais beaucoup plus rapide et comportant beaucoup moins de pertes d'alevins. Au lieu d'être une opération coûteuse et spectaculaire et même un peu risquée lors des atterrissages près des lacs en des endroits mal ou non balisés, elle devient une opération pratique, économique et sans danger. Il suffit de bien la préparer et d'approvisionner la base de départ pour éviter les temps morts.

En ce qui nous concerne, nous obtenons de l'Administration le crédit nécessaire pour effectuer normalement cette opération chaque année.

Précisons enfin que, tout comme le sabre de M. Prudhomme qui pouvait servir à défendre les institutions et au besoin aussi à les combattre, l'hélicoptère sert à aleviner les lacs de montagne et aussi à porter sur place les pêcheurs. Pendant l'été 1958, à plusieurs reprises, des pêcheurs ont loué des hélicoptères à la $\mathrm{S}$. C. E. T. A. pour se faire transporter sans fatigue près des lacs de haute altitude alevinés les années précédentes. Les lacs de montagne pyrénéens peu fréquentés et peu pêchés autrefois, le sont de plus en plus aujourd'hui. C'est ainsi qu'en Juin 1958, nous avons compté un jour plus de 60 pêcheurs aux lacs de Grésiolles et Caderolles, situés à 2 heures de marche de la route. L'hélicoptère va donc permettre une fréquentation plus grande encore de ces lacs tout en nous fournissant, heureusement, les moyens d'alevinage nécessaires au maintien de leur richesse piscicole. 
La liste des lacs est tirée de Gaurier, vériflée et rectiflée.

L.es lacs sont énumérés de l'Ouest à l'Est.

Abréviations : tc : Truite commune. - ta : Truite arc-en-ciel. - st : Saumon de fontaine. - oc : Omble-Chevalier. - $\mathrm{v}$ : Véron. - cn : Cristivomer namaycush. - th : Tanche.

N. $B$. - Les lacs marqués tc sont des lacs où la Truite commune existait naturellement, sauf mention dans la colonne observations.

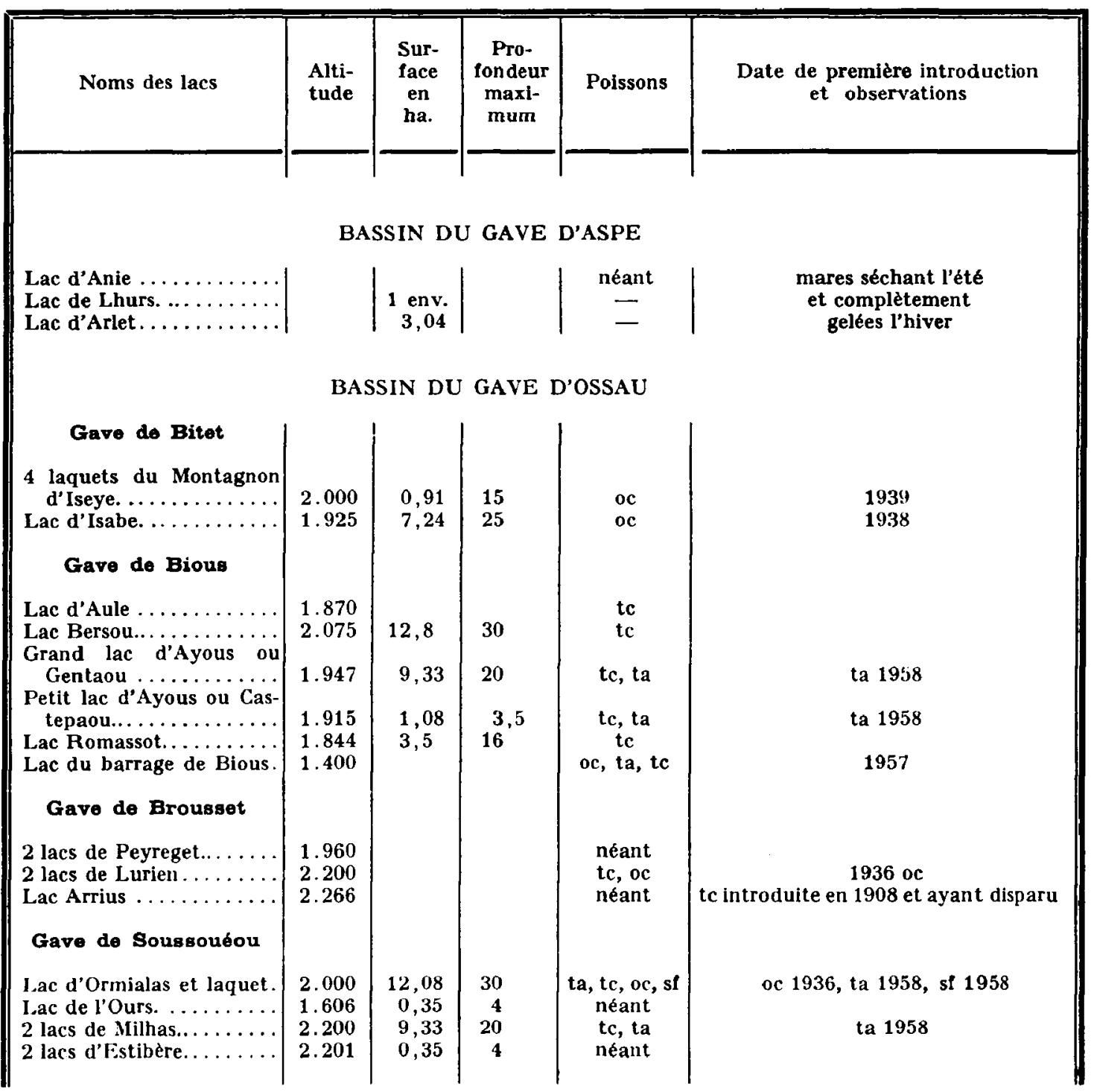




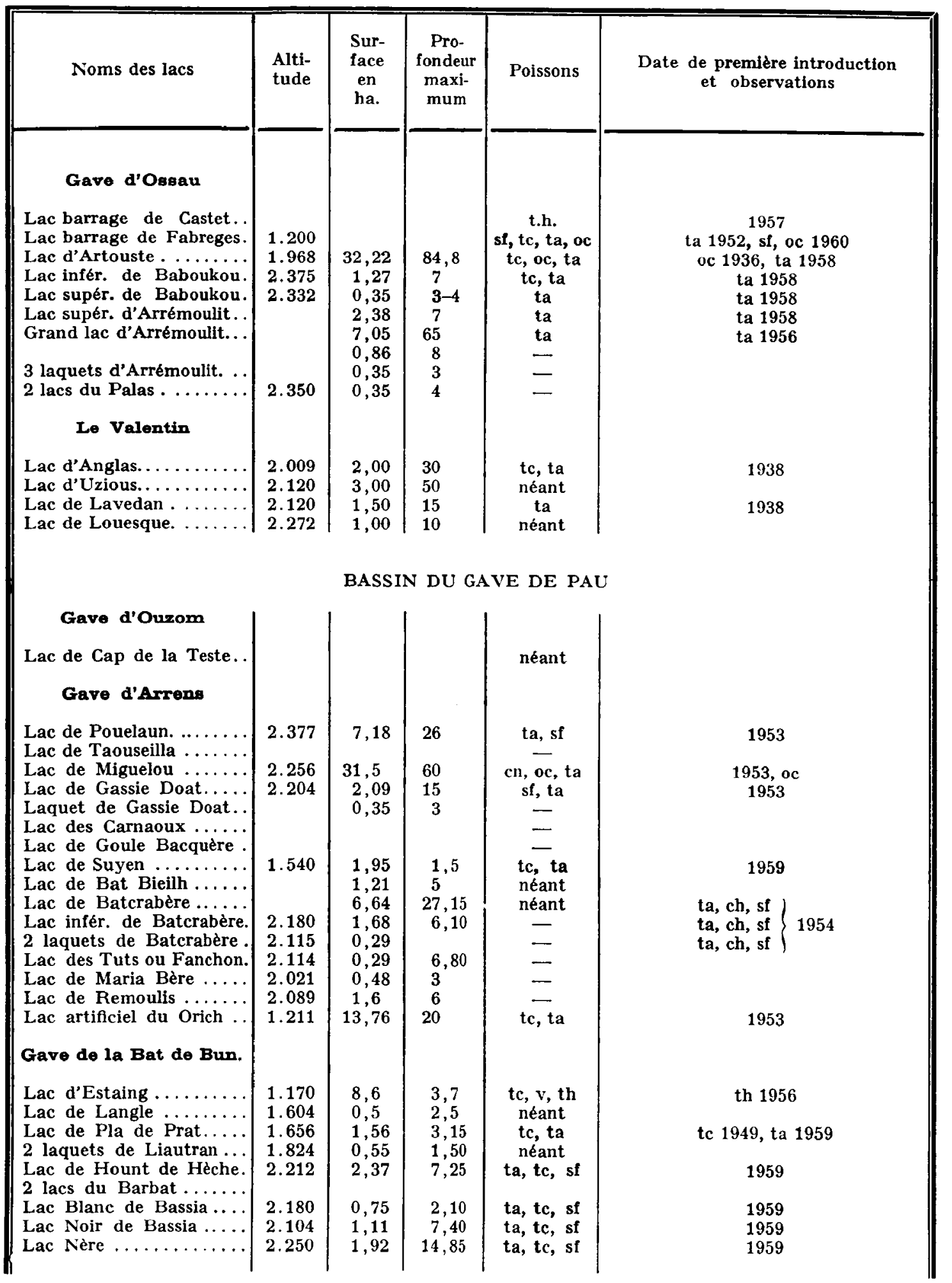




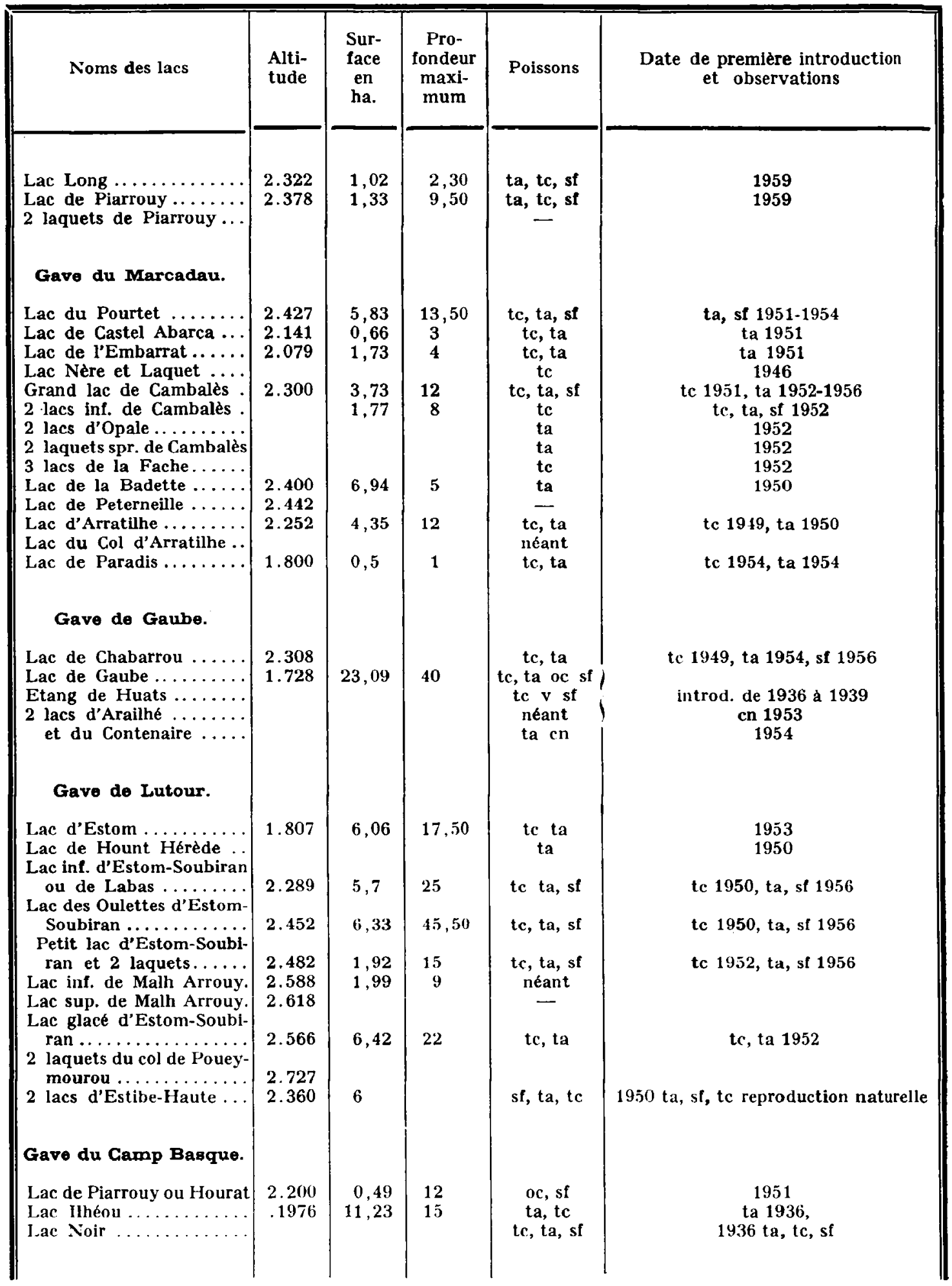




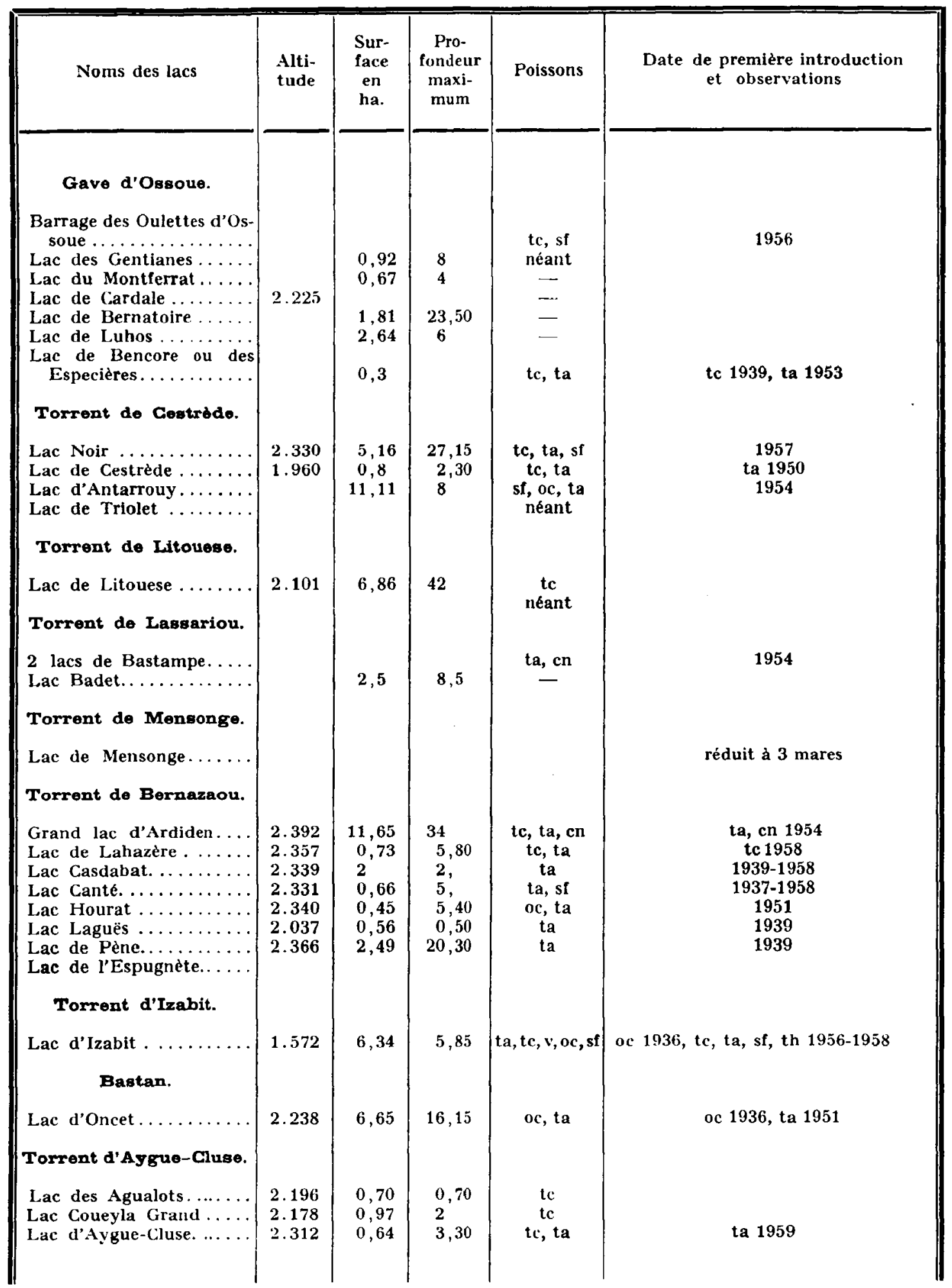




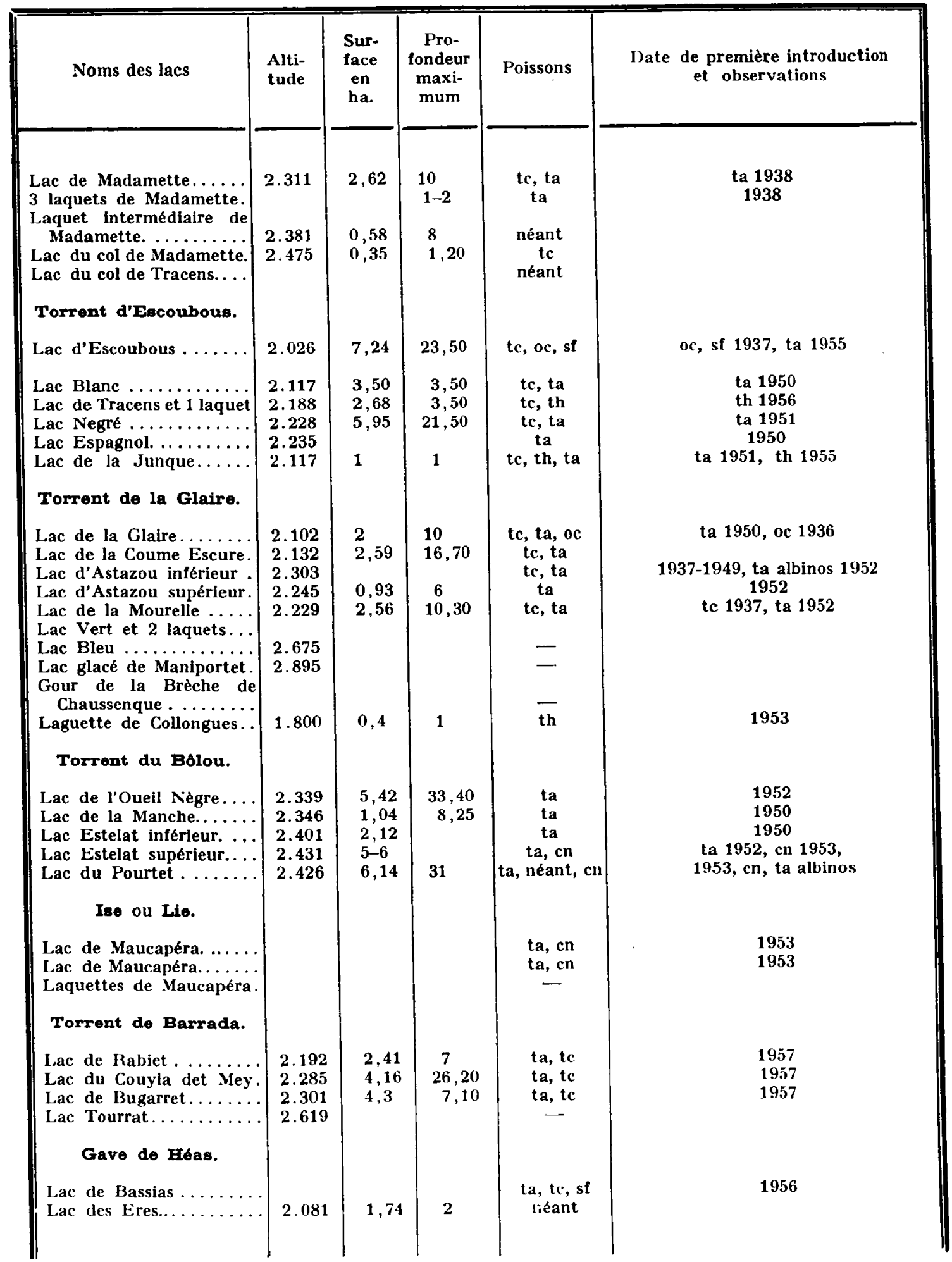




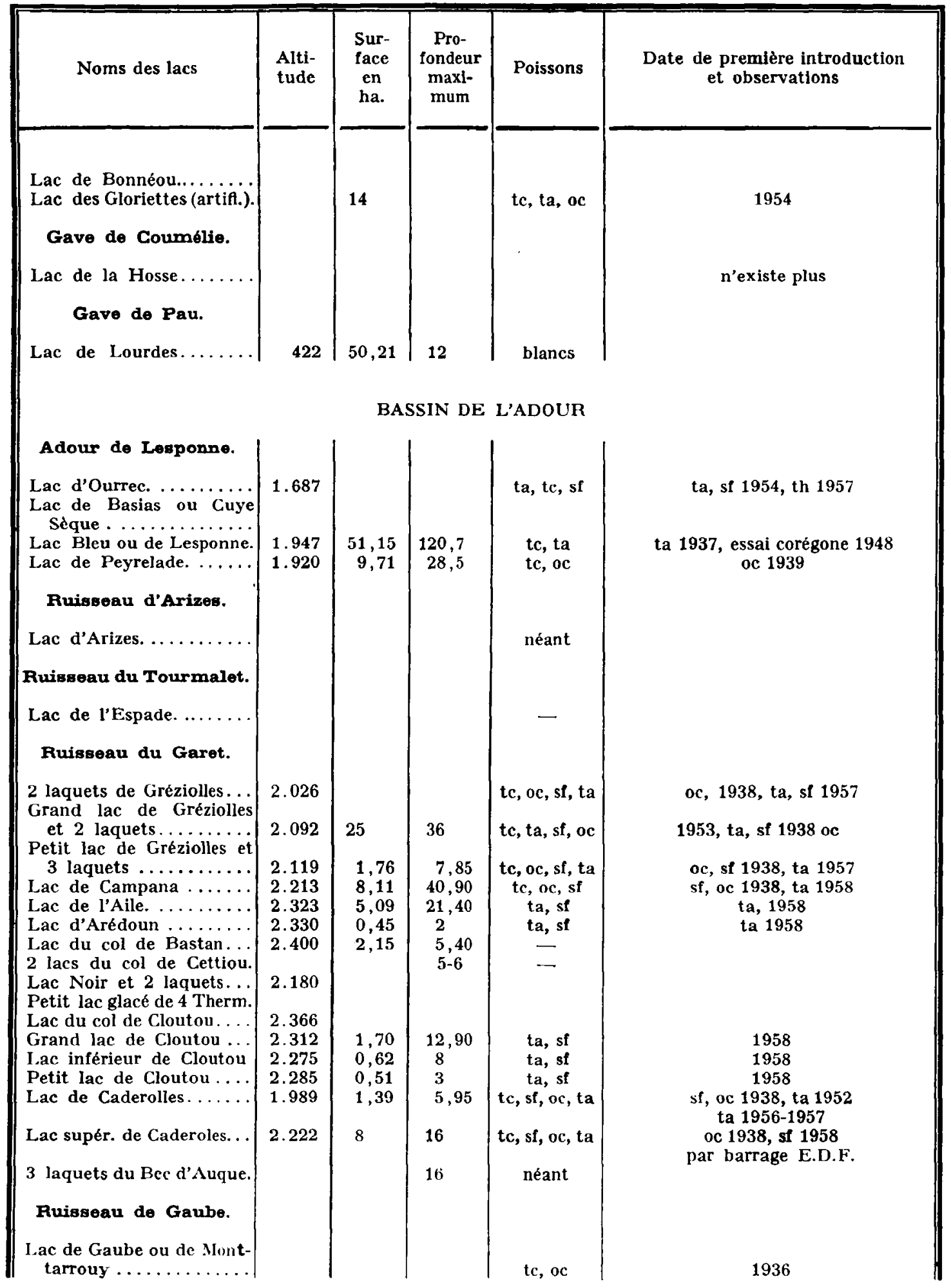




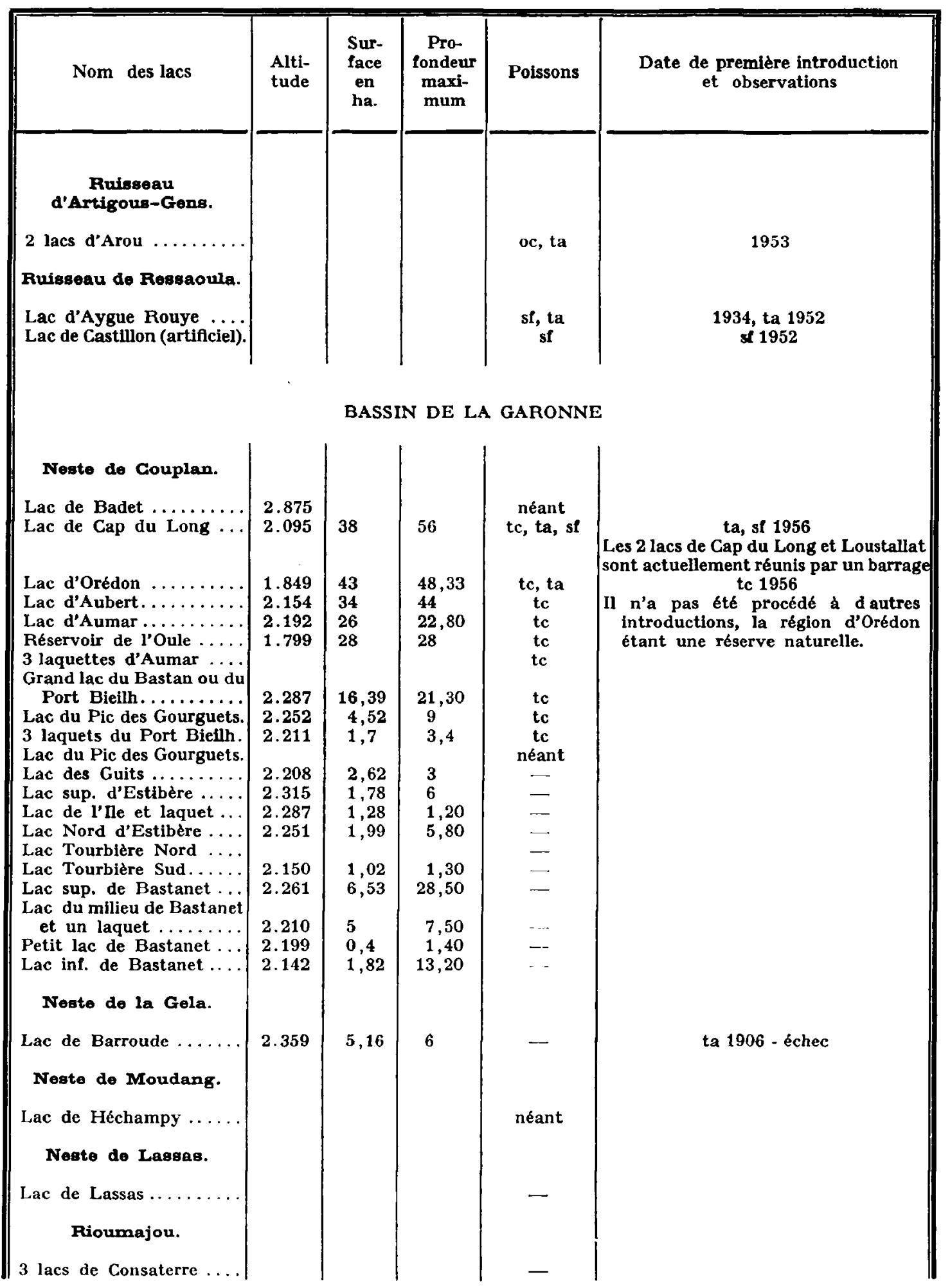




\begin{tabular}{|c|c|c|c|c|c|}
\hline Noms des lacs & $\begin{array}{l}\text { Alti- } \\
\text { tude }\end{array}$ & $\begin{array}{l}\text { Sur- } \\
\text { face } \\
\text { en } \\
\text { ha. }\end{array}$ & $\begin{array}{c}\text { Pro- } \\
\text { fondeur } \\
\text { maxi- } \\
\text { mum }\end{array}$ & Poíssons & $\begin{array}{c}\text { Date de première introduction } \\
\text { et observations }\end{array}$ \\
\hline 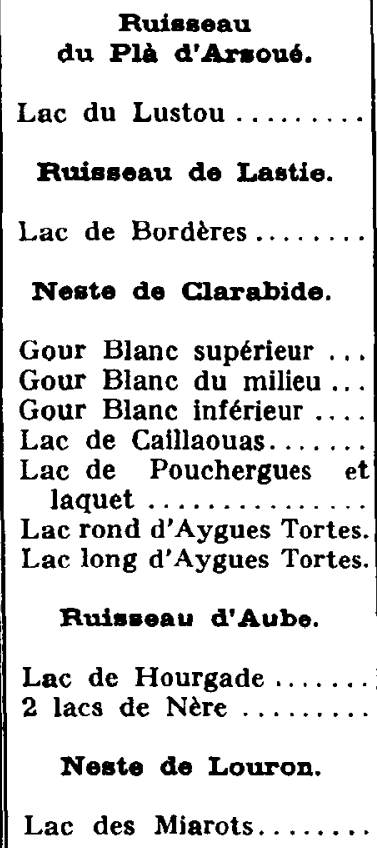 & $\begin{array}{l}2.368 \\
\\
1.763 \\
\\
2.560 \\
2.524 \\
2.395 \\
2.155 \\
2.165 \\
2.275 \\
2.265\end{array}$ & $\begin{array}{l}4,31 \\
6,31 \\
4,51 \\
8,49\end{array}$ & $\begin{array}{l}8,50 \\
14,50 \\
100 \\
32,20\end{array}$ & $\begin{array}{c}\text { oc, tc, ta } \\
\text { néant } \\
-\overline{-} \text { oc, tc, ta } \\
\text { tc } \\
\text { néant } \\
- \\
\text { ta, oc } \\
\text { ta, oc }\end{array}$ & oc 1938 , tc, ta 1958 \\
\hline
\end{tabular}

